\title{
Mid-infrared Transmission Properties of Step index and Large Mode Area ZnSe Microstructured Optical Fibers
}

\author{
N. Healy ${ }^{1}$, J.R. Sparks ${ }^{2}$, Rongrui He ${ }^{2}$, M.N. Petrovich ${ }^{1}$, P.J.A. Sazio ${ }^{1}$, J.V. Badding ${ }^{2}$ and A.C. Peacock ${ }^{1}$ \\ ${ }^{I}$ Optoelectronic Research Centre, University of Southampton, Southampton SO17 1BJ, UK \\ nvh@orc.soton.ac.uk \\ ${ }^{2}$ Department of Chemistry and Materials Research Institute, Pennsylvania State University, 16802 PA, USA
}

\begin{abstract}
ZnSe microstructured fibers have been designed and fabricated using silica capillaries and an air-silica photonic band-gap optical fiber as high-pressure microfluidic templates for semiconductor growth via chemical fluid deposition. We examine their transmission properties over a wide spectral range.

OCIS codes: (060.2280) Fiber design and fabrication; (060.2290) Fiber materials; (160.6000) Semiconductor materials; (190.4370) Nonlinear optics, fibers
\end{abstract}

\section{Introduction}

Integration of semiconductors into optical fibers is greatly expanding the range of extreme aspect ratio electronic/photonic devices that can be fabricated. Although methods have been developed to fabricate fibers incorporating crystalline unary semiconductors and amorphous compound semiconductors [1,2], a key challenge is to realize low optical loss crystalline compound semiconductor fibers. Such compound semiconductors can have properties not found in typical unary semiconductors, such as high second order non-linear optical coefficients that allow for efficient second harmonic generation and direct bandgaps that allow for efficient generation of light. Furthermore, compound semiconductors can have higher carrier mobilities than technologically relevant unary semiconductors and be optically transparent over a wider wavelength range. An additional advantage of crystalline compound semiconductors is their ability to host transition metal ions that can serve as optical gain media; $\mathrm{Cr}^{2+}$ doped $\mathrm{ZnSe}$ functions as an excellent gain medium in the 2 to $3 \mu \mathrm{m}$ region of the infrared spectrum, for example [3]. Fiber waveguiding gain media can have properties superior to those of bulk gain media, such as relative insensitivity to troublesome thermal lensing effects that limit power handling and extended interaction lengths between light and the gain media.

\section{Fiber fabrication and optical characterization}

The high pressure chemical fluid (CFD) technique we have developed $[1,4]$ is low cost and flexible in that it can be modified to fill a range of capillaries with differing core dimensions, while high production rates can be obtained by parallel fabrication of multiple fibers in a single deposition. It can also be extended to fill the large number of micro/nanoscale pores in microstructured optical fibers (MOFs), providing additional geometrical design flexibility to enhance the potential application base of the fiber devices [5]. A further advantage of this fabrication approach is that the surface of the deposited material is defined by the pristine smoothness of the capillary surfaces which have been shown to have a root-mean-square (RMS) roughness of only $0.1 \mathrm{~nm}$ [6]. The resulting semiconductor fibers fabricated via CFD further benefit from the properties of the silica templates which are robust, flexible, and are compatible with existing optical fiber infrastructures.

Fig. 1a shows a SEM image of a ZnSe optical fiber, fabricated by filling the pore of a silica capillary with a $\sim 15 \mu \mathrm{m}$ internal diameter, from which the crystalline grain structure can be seen. The existence of a small $(\sim 500 \mathrm{~nm})$ hole is a result of the necessity of the byproducts from the chemical deposition to flow out of the fiber and can be reduced in optimized depositions by using higher pressures. These fibers can be mounted in thicker capillaries and their end faces polished for optimal coupling into the core (Fig. 1b). Raman and photoluminescence measurements on the core material verify the high quality of the deposited $\mathrm{ZnSe}$ [4] which suggest that the fibers will function well as low loss waveguides. Measurements of the transmission loss this ZnSe fiber over the wavelength range of 500 to 2500 $\mathrm{nm}$ confirm this expectation (Fig. 1c). The losses decrease with increasing wavelength and become as low as $0.5 \mathrm{~dB} / \mathrm{cm}$ in the 2000 to $2400 \mathrm{~nm}$ mid-IR region. The loss as a function of the wavelength can be fitted from 540 to $2400 \mathrm{~nm}$ by a power law with an exponent of $-3.9 \pm 0.2$. This behavior is well matched to the wavelength dependence that is characteristic of Rayleigh scattering $\left(\lambda^{4}\right)$, suggesting that scattering from bulk refractive index inhomogeneities (possibly due to grain boundaries) in the material is the dominant loss mechanism. We will present 
additional data on this step index ZnSe fiber that extend our measurements further into the mid-IR range to investigate the predicted dominant Rayleigh scattering behavior.
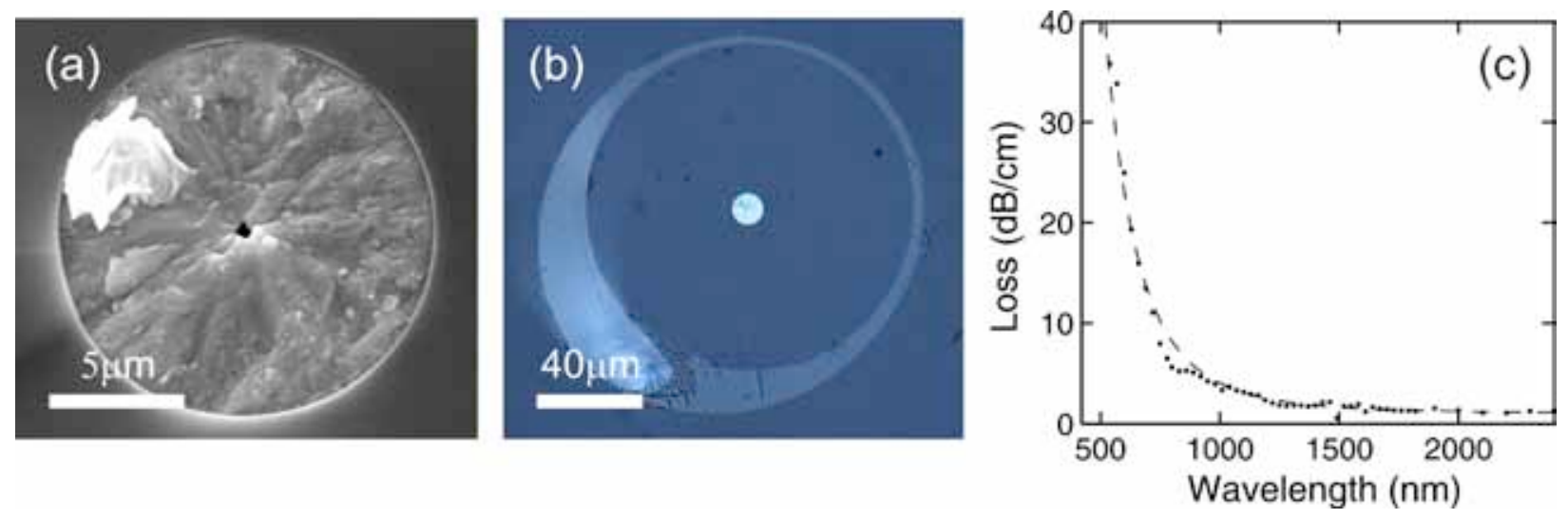

Figure 1. (a) SEM image of a silica step index fiber with a $15 \mu \mathrm{m}$ diameter ZnSe core. (b) Optical micrograph of the silica/ZnSe fiber mounted in a larger capillary for end-face polishing. (c) Transmission loss of the fiber as a function of wavelength, dashed line is a $\lambda^{-3.9}$ fit.

We have also built on previous work [5] to demonstrate a novel ZnSe MOF (Fig. 2). The fiber was fabricated by filling the air holes of a hollow core silica photonic bandgap (PBG) fiber, which has a core diameter of $\sim 9 \mu \mathrm{m}$, with the semiconductor material via the high pressure CFD process. Compared to the step index fiber shown in Fig. 1, the $\mathrm{ZnSe}$ MOF in Fig. 2a has a cladding with a higher effective index than pure silica so that we can selectively couple into a reduced number of core modes. As we will discuss during the presentation, this $\mathrm{ZnSe}$ fiber is effectively dual mode over a wide wavelength range (Fig. 2b-c), though with further optimization of the PBGF template and deposited material it should be possible to design a $\mathrm{ZnSe} \mathrm{MOF}$ for effective single mode operation [7]. Initial transmission measurements in this fiber at $1550 \mathrm{~nm}$ show it has a loss of $\sim 1.9 \mathrm{~dB} / \mathrm{cm}$ and we will further investigate its mid-infrared optical characteristics. This slightly higher loss value than in the step index fiber is likely to be due both to the enhanced interaction of the core guided light with the high index microstructured cladding and the nonoptimized material deposition.
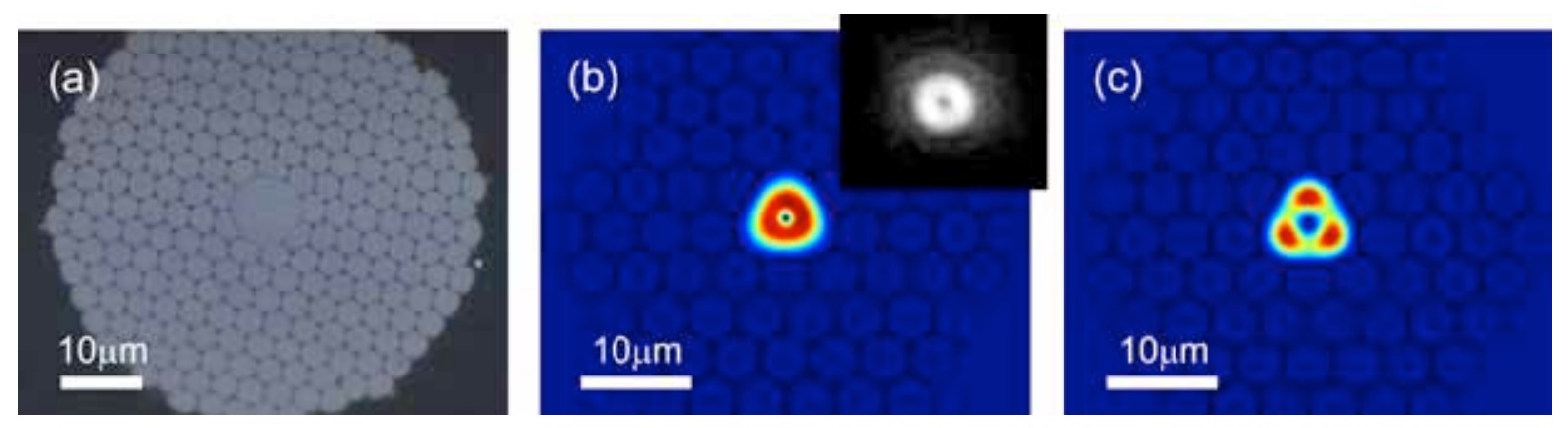

Figure 2. (a) Optical micrograph of a ZnSe MOF. Although not visible in this image, the core and cladding rods have very small $(<500 \mathrm{~nm})$ holes in the center, similar to Fig. 1(a). Finite element simulations of the ZnSe MOF showing the two main core modes where (b) is the fundamental and (c) is the second order mode calculated at $1550 \mathrm{~nm}$. Inset in (b) shows the actual fundamental guided mode at $1550 \mathrm{~nm}$ imaged using an Electrophysics MicronViewer 7290A infrared camera.

\section{Conclusions}

We have demonstrated integration of crystalline ZnSe into optical fibers to form low loss mid-IR waveguides that exploit the superior properties of crystalline compound semiconductors for the fabrication of photonic devices such as fiber amplifiers and lasers, as well as for non-linear optical processing. We have shown that extension to deposition in ordered arrays of pores in MOF templates is straightforward and allows for a reduction in the number 
of modes that can be excited in the core, as previously demonstrated for silicon MOFs [5]. The properties of an optimized ZnSe MOF will make it a suitable candidate for the realization of effectively single mode, high power and/or multiwavelength mid-IR in-fiber devices. We note that the approach to fabricating ZnSe core optical fibers via CFD reported here should be applicable to a broad range of compound semiconductors via assessment/development of suitable precursor chemistries. The range of high performance electronic and optoelectronic function that could then be integrated into optical fiber devices extends far beyond what is currently possible with amorphous materials and crystalline unary semiconductors.

\section{References}

[1] P. J. A. Sazio, A. Amezcua-Correa, C. E. Finlayson, J. R. Hayes, T. J. Scheidemantel, N. F. Baril, B. R. Jackson, D.-J. Won, F. Zhang, E. R. Margine, V. Gopalan, V. H. Crespi, and J. V. Badding, "Microstructured Optical Fibers as High-Pressure Microfluidic Reactors," Science 311, 1583-1586 (2006).

[2] J. Ballato, T. Hawkins, P. Foy, R. Stolen, B. Kokuoz, M. Ellison, C. McMillen, J. Reppert, A. M. Rao, M. Daw, S. Sharma, R. Shori, O. Stafsudd, R. R. Rice, and D. R. Powers, “Silicon optical fiber,” Opt. Express 16, 18675-18683 (2008).

[3] I. T. Sorokina, "Cr ${ }^{2+}$-doped II-VI materials for lasers and nonlinear optics," Opt. Mater. 26, 395-412 (2004).

[4] J. R. Sparks, R. He, N. Healy, M. Krishnamurthi, A. C. Peacock, P. J. A. Sazio, V. Gopalan, and J. V. Badding, "Zinc Selenide Optical Fibers," Adv. Mater. 23, 1647-1651 (2011).

[5] N. Healy, J.R. Sparks, M.N. Petrovich, P.J.A. Sazio, J.V. Badding and A.C. Peacock, "Large mode area silicon microstructured fiber with robust dual mode guidance," Opt. Express 17(20) 18076-18082 (2009).

[6] P. J. Roberts, F. Couny, H. Sabert, B. J. Mangan, D. P. Williams, L. Farr, M. W. Mason, A. Tomlinson, T. A. Birks, J. C. Knight, and P. St. J. Russell, "Ultimate low loss of hollow-core photonic crystal fibres," Opt. Express 13, 236-244 (2005).

[7] N. Vukovic, N. Healy, and A.C. Peacock, "Guiding properties of large mode area silicon microstructured fibers: a route to effective single mode operation," JOSA B 28, 1529-1533 (2011). 